\title{
Extracción de $\mathrm{Ag}(\mathrm{I})$ en medio cloruro sódico con sulfuro de tri-isobutil fösfina ${ }^{(\bullet)}$

\author{
F.J. Alguacil ${ }^{(*)}$ y S. Martínez $\left.{ }^{*}\right)$
}

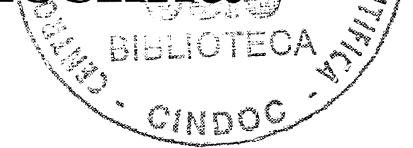

\begin{abstract}
Resumen Se estudia la extracción de $\mathrm{Ag}(\mathrm{I})$ de disoluciones en medio cloruro sódico concentrado mediante el agente de extracción sulfuro de tri-isobutil fosfina. La plata se extrae en este medio cloruro sódico mediante una reacción de solvatación en la que la estequiometría de la especie extraida mantiene la relación molar agente de extracción: Ag igual a 2. La extracción de plata mediante este agente de reextracción responde a reacciones exotérmicas, independientemente del diluyente orgánico y del medio acuoso empleado. El sistema de extracción es selectivo frente a la presencia de cobre y cinc en el medio acuoso. La plata puede extraerse mediante disoluciones de tiosulfato sódico. Se han obtenido los valores de $\log K_{\text {ext }} \Delta G^{\circ}$ y $\Delta S^{\circ}$ a $25^{\circ} \mathrm{C}$ para el sistema de extracción $\mathrm{Ag}(\mathrm{I})$-STIBF en disoluciones acuosas en medio cloruro sódico concentrado.
\end{abstract}

Palabras clave: Extracción con disolventes. $\operatorname{Ag}(\mathbf{I})$. Disoluciones de cloruro sódico. Sulfuro de triisobutil fosfina.

\section{Extraction of $\operatorname{Ag}(\mathrm{I})$ with tri-isobutyl phosphine sulphide from sodium chloride media}

\begin{abstract}
The solvent extraction of $\mathrm{Ag}(\mathrm{I})$ from concentrated sodium chloride solutions by the extractant triisobutyl phosphine sulphide is studied. Silver is extracted from this sodium chloride media by means of a solvation reaction, the stoichiometry of the extracted species is related to an extractant: Ag molar relationship of 2 . Silver extraction by menas of TIBFS is related to exothermic extraction reactions this behaviour independent both of the aqueous media and the organic diluent. The extraction system is selective with respect to the presence of $\mathrm{Cu}$ and $\mathrm{Zn}$ in the aqueous phase. Silver is stripped by sodium tiosulphate solutions. Values of $\log K_{\mathrm{ext}}, \Delta G^{\circ}$ and $\Delta S^{\circ}$ at $25^{\circ} \mathrm{C}$ for the present extraction system were also given.
\end{abstract}

Keywords: Solvent extraction. Ag(I). Sodium chloride solutions. Tri-isobutyl phosphine sulphide.

\section{INTRODUCCIÓN}

Actualmente, la aplicación de la extracción con disolventes como operación unitaria en los procesos hidrometalúrgicos, se extiende a la recuperación de una serie de metales de disoluciones que provienen del tratamiento de distintos materiales de partida.

Asimismo, existe una mayor utilización de la hidrometalurgia en medio cloruro para el tratamiento de distintas materias primas, especialmente sulfuradas, lo que ha llevado a que también se preste mayor atención a los procesos de recuperación de

$(\bullet$ Trabajo recibido el día 16 de enero de 1995.

${ }^{*}$ Centro Nacional de Investigaciones Metalúrgicas. (CSIC). Avda. de Gregorio del Amo, 8. 28040-Madrid (España). los metales preciosos que, generalmente, se encuentran presentes en estas materias primas y tienden a formar una serie de complejos estables y solubles en estas disoluciones de lixiviación, con lo que se facilita, en principio, su posterior recuperación.

La eficacia de los procesos de extracción con disolventes aplicados a la recuperación y beneficio de los metales preciosos, contenidos en estas disoluciones en medio cloruro, se ha asociado principalmente a agentes de extracción neutros que contengan átomos de azufre, oxígeno y/o fósforo en su molécula, además de las aminas que tienden a extraer a estos metales mediante mecanismos de intercambio aniónico (1-4).

En el caso de los agentes de extracción neutros o por solvatación que contienen átomos de azufre, su 
aplicación en este campo se debe a la consideración de base blanda que presentan estos reactivos, en los que el átomo de azufre actúa como donador de electrones, capaces de formar complejos estables con ácidos blandos, como, por ejemplo, la $\mathrm{Ag}(\mathrm{I}) \mathrm{y}$, por lo tanto, extraer estos metales de las disoluciones acuosas.

Pese a los datos que existen sobre la extracción de metales preciosos en distintos medios acuosos, en el caso de la plata no se dispone del mismo grado de información, sobre todo en lo que se refiere a la interpretación de los mecanismos de extracción de este metal de las diferentes disoluciones acuosas que lo contienen $(3,5$ y 6$)$.

En el presente trabajo se estudia la extracción de $\operatorname{Ag}(\mathrm{I})$, en medio cloruro sódico, mediante el agente de extracción sulfuro de tri-isobutil fosfina, con el fin de contribuir al conocimiento sobre el comportamiento químico de estos sistemas.

\section{PARTE EXPERIMENTAL}

El sulfuro de tri-isobutil fosfina (en adelante STIBF), se obtuvo del agente de extracción de nombre comercial Cyanex 471X, cedido generosamente por American Cyanamid Co. Este reactivo, que se purificó por recristalización de disoluciones etanol-agua, es un sólido blanquecino que funde en el intervalo $58-59{ }^{\circ} \mathrm{C}$ y presenta un peso molecular de aproximadamente 237. La estructura química es $\mathrm{R}_{3} \mathrm{P}=\mathrm{S}$, donde $\mathrm{R}$ representa al grupo isobutil (7).

Las concentraciones de plata empleadas responden a valores típicos obtenidos de disoluciones de lixiviación (8), mientras que en el caso de las fases orgánicas se ensayaron distintas concentraciones del agente de extracción de forma que siempre se lograra la solubilización adecuada de dicho agente en el correspondiente diluyente orgánico.

Los diluyentes orgánicos queroseno y Solvesso 100 se obtuvieron, respectivamente, de CAMPSA y Exxon Chemical Iberia. Sus características principales se muestran en la tabla I. El resto de los reactivos químicos empleados fue de calidad R.A.

Los ensayos de extracción se llevaron a cabo en embudos de separación termostatizados a la temperatura requerida y provistos de agitación mecánica.
Se emplearon, en general, volúmenes iguales de las respectivas fases orgánicas y acuosas.

El análisis de los metales se realizó mediante espectrofotometría de absorción atómica.

A lo largo del trabajo, el valor del coeficiente de distribución del metal, representado por $D$, se calculó según la relación:

$$
D_{\mathrm{Me}}=\frac{[M e]_{\mathrm{org}}}{[M e]_{\mathrm{ac}}}
$$

donde $[\mathrm{Me}]_{\text {org }}$ y $[\mathrm{Me}]_{\mathrm{ac}}$ representan las concentraciones analíticas de los metales en cada fase después de poner en contacto las mismas.

A partir de los datos experimentales obtenidos en el presente trabajo, y teniendo en cuenta la relación entre el coeficiente de distribución y la temperatura (9), se calcularon los correspondientes valores del cambio de entalpía para cada sistema de extracción.

\section{RESULTADOS Y DISCUSIÓN}

\subsection{Influencia del tiempo de equilibrado}

El estudio experimental se llevó a cabo con una fase orgánica de $50 \mathrm{~g} / \mathrm{l}$ del agente de extracción en queroseno y una fase acuosa de $0,2 \mathrm{~g} / \mathrm{l} \mathrm{Ag}(\mathrm{I})$ en un medio $\mathrm{NaCl} 4 \mathrm{M}$. La temperatura fue de $25{ }^{\circ} \mathrm{C}$, mientras que el tiempo de equilibrado se varió entre 2,5 min y 2 h de agitación.

El equilibrio de extracción se alcanza a partir de los 10 min de agitación, siendo el cambio del porcentaje de extracción de $\operatorname{Ag}(\mathrm{I})$ del orden del $5 \%$ para tiempos de contacto comprendidos entre 2,5 y 10 min, no aumentando el contenido de plata en la fase orgánica para tiempos de contacto más prolongados; este hecho indica que la estequiometría de la especie extraída no cambia con estos mayores tiempos de equilibrado. Resultados similares se obtienen con otras concentraciones iniciales de plata y/o STIBF.

\subsection{Influencia de la temperatura}

El estudio del efecto de esta variable sobre la extracción de plata se ha realizado con fases

TABLA I.- Características de los diluyentes

TABLE I.- Characteristics of the diluents

\begin{tabular}{|l|c|c|c|c|}
\hline Diluyente & $\begin{array}{c}\text { Punto de } \\
\text { ebullición, }{ }^{\circ} \mathrm{C}\end{array}$ & $\begin{array}{c}\text { Punto de } \\
\text { inflamación, }{ }^{\circ} \mathrm{C}\end{array}$ & $\begin{array}{c}\text { Densidad, } \\
\mathrm{kg} / \mathrm{m}^{3}\end{array}$ & $\begin{array}{c}\text { Compuestos } \\
\text { aromáticos, } \%\end{array}$ \\
\hline Queroseno & $>140$ & 48 & 780 & 20 \\
Solvesso 100 & $162-178$ & 45 & 878 & $>99$ \\
\hline
\end{tabular}


orgánicas de 25 ó $100 \mathrm{~g} / \mathrm{l}$ STIBF en queroseno y fases acuosas de 0,05 ó $0,2 \mathrm{~g} / \mathrm{l} \mathrm{Ag}(\mathrm{I})$ en un medio $\mathrm{NaCl} 4 \mathrm{M}$. El tiempo de equilibrado fue de $10 \mathrm{~min}$.

La tabla II muestra los resultados obtenidos; se observa que el aumento de la temperatura a la que se realiza la extracción disminuye el tanto por ciento de plata extraída por este agente.

A partir de la figura 1 se deduce que $\Delta H^{\circ}=-40,9$ $\mathrm{kJ} / \mathrm{mol}$, indicativo del carácter exotérmico de la reacción de extracción.

\subsection{Influencia del diluyente de la fase orgánica}

En general, el diluyente de la fase orgánica se emplea para adecuar las condiciones de trabajo de cada sistema de extracción en particular. Sin embargo, el diluyente de la fase orgánica puede afectar o influir en las propiedades de extracción de un determinado agente de extracción, siendo, además, muy difícil generalizar en este punto.

En el presente trabajo se ha evaluado el comportamiento del STIBF en la extracción de $\mathrm{Ag}(\mathrm{I})$ frente a dos diluyentes orgánicos, que se diferencian principalmente en su composición química, uno, el queroseno, fundamentalmente alifático, y otro, el Solvesso 100 , que es aromático.

El estudio experimental se llevó a cabo con disoluciones de $100 \mathrm{~g} / \mathrm{l} \mathrm{STIBF}$ en cada uno de los dos diluyentes mencionados. La fase acuosa contenía $0,05 \mathrm{~g} / \mathrm{l} \mathrm{Ag}(\mathrm{I})$ en un medio $\mathrm{NaCl} 4 \mathrm{M}$. El tiempo de equilibrado fue de $10 \mathrm{~min}$, ensayándose, al mismo tiempo, a temperaturas comprendidas entre 20 y $70^{\circ} \mathrm{C}$.

Los resultados obtenidos se muestran en la figura 2, que representa la variación de $\log D_{\mathrm{Ag}}$ frente a $1.000 / T$ para los dos sistemas. Se deduce que el cambio de diluyente afecta a la extracción de $\mathrm{Ag}(\mathrm{I})$ por el sulfuro de tri-isobutil fosfina, para cada temperatura ensayada se obtienen mejores resultados

TABLA II.- Influencia de la temperatura en la extracción de $\mathrm{Ag}(\mathrm{I})$ mediante STIBF

TABLE II.- Influence of temperature on $\mathrm{Ag}(\mathrm{I})$ extraction by TIBPS

\begin{tabular}{|c|c|c|c|}
\hline $\begin{array}{c}\text { Tempe- } \\
\text { ratura, }{ }^{\circ} \mathrm{C}\end{array}$ & $\begin{array}{c}\text { Ag inicial, } \\
\mathrm{mol} / \mathrm{l}\end{array}$ & $\begin{array}{c}\text { STIBF, } \\
\mathrm{g} / \mathrm{l}\end{array}$ & $\begin{array}{c}\text { Extrac. } \\
\mathrm{Ag}(\mathrm{I}), \%\end{array}$ \\
\hline 20 & $4,64 \cdot 10^{-4}$ & 100 & 97,0 \\
30 & $4,64 \cdot 10^{-4}$ & 100 & 93,3 \\
40 & $4,64 \cdot 10^{-4}$ & 100 & 88,0 \\
50 & $4,64 \cdot 10^{-4}$ & 100 & 82,5 \\
60 & $4,64 \cdot 10^{-4}$ & 100 & 78,1 \\
70 & $4,64 \cdot 10^{-4}$ & 100 & 73,2 \\
20 & $1,85 \cdot 10^{-3}$ & 25 & 52,5 \\
40 & $1,85 \cdot 10^{-3}$ & 25 & 43,0 \\
60 & $1,85 \cdot 10^{-3}$ & 25 & 33,6 \\
\hline
\end{tabular}

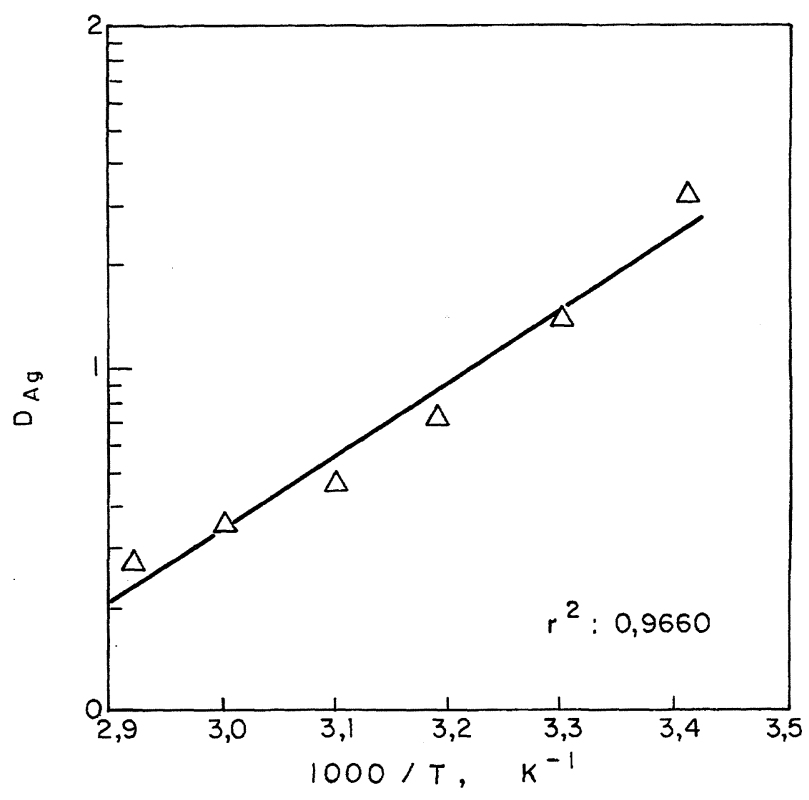

FIG. 1.- Variación de $\log D_{\mathrm{Ag}}$ frente a 1.000/T para la extracción de $\operatorname{Ag}(\mathrm{I})$ mediante STIBF. Fase acuosa: 0,05 g/l $\mathrm{Ag}(\mathrm{I})$ en medio $\mathrm{NaCl} 4 \mathrm{M}$. Fase orgánica: $100 \mathrm{~g} / \mathrm{l} \mathrm{STIBF}$ en queroseno.

FIG. 1.-Arrhenius plot for the extraction of $A g(I)$ by TIBPS. Aqueous phase: $0.05 \mathrm{~g} / \mathrm{l} \mathrm{Ag}(\mathrm{I})$ in $\mathrm{NaCl} 4 \mathrm{M}$ media. Organic phase: $100 \mathrm{~g} / \mathrm{l}$ TIBPS in kerosene.

de extracción cuando se emplea Solvesso 100 como diluyente, siendo la diferencia más notoria a medida que aumenta la temperatura de extracción, aunque cuando se emplea Solvesso 100 también disminuye la extracción del metal al aumentar la temperatura de extracción.

A partir de esta figura se obtiene que, para el sistema que emplea Solvesso $100, \Delta H^{\circ}=-31,0 \mathrm{~kJ} / \mathrm{mol}$, presentando la reacción de extracción, también, carácter exotérmico.

\subsection{Influencia del cambio del medio acuoso}

Se han llevado a cabo una serie de ensayos para estudiar el efecto que sobre la extracción de plata provoca un aumento de la acidez del medio acuoso $\mathrm{y}$, en último extremo, el cambio total del cloruro sódico por ácido clorhídrico en la disolución acuosa.

En la tabla III se muestran las condiciones experimentales y los resultados obtenidos en esta serie de ensayos. El aumento de la concentración de ácido clorhídrico en la disolución acuosa tiende a disminuir el tanto por ciento de plata extraída en la fase orgánica, aunque, para una concentración inicial de $\mathrm{HCl} 1 \mathrm{M}$ la extracción del metal vuelve a aumentar ligeramente con respecto al caso en el que el contenido de este ácido es menor. En este medio $\mathrm{NaCl} 4 \mathrm{M}$, cuando el contenido del ácido es superior 


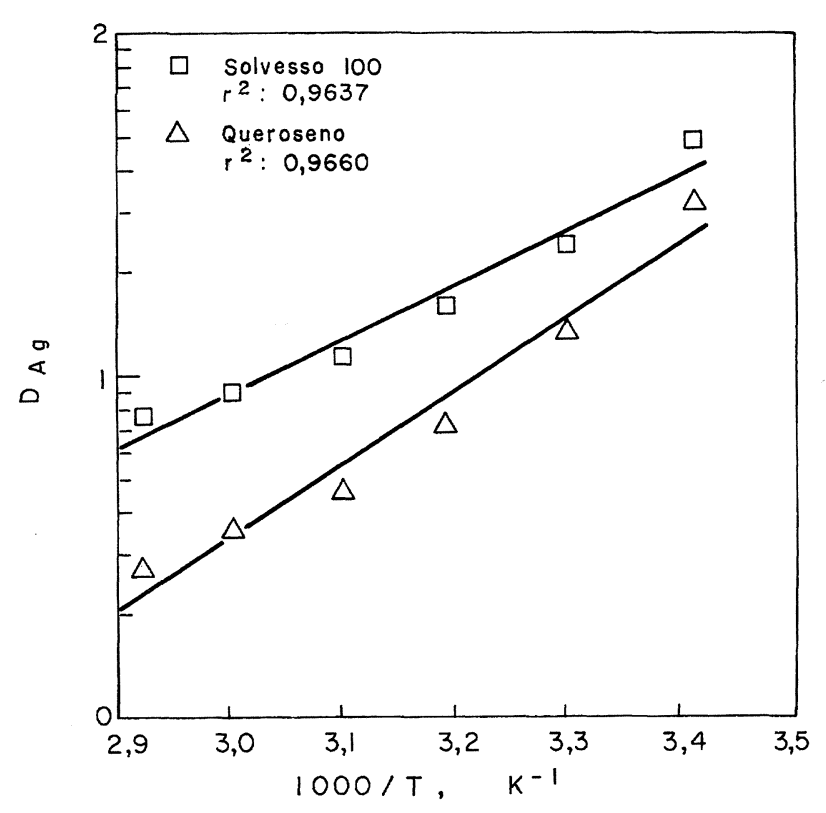

FIG. 2.- Influencia de la temperatura sobre la extracción de $\operatorname{Ag}(\mathrm{I})$ mediante STIBF. Fase acuosa: $0,05 \mathrm{~g} / \mathrm{l} \mathrm{Ag}(\mathrm{I})$ en medio $\mathrm{NaCl} 4 \mathrm{M}$. Fase orgánica:

$100 \mathrm{~g} / \mathrm{l}$ STIBF en Solvesso 100 o queroseno.

FIG. 2.-Arrhenius plot for the extraction of $\mathrm{Ag}(I)$ by TIBPS. Aqueous phase: $0.05 \mathrm{~g} / \mathrm{l} \mathrm{Ag}(\mathrm{I})$ in $\mathrm{NaCl}$ $4 M$ media. Organic phase: $100 \mathrm{~g} / \mathrm{l}$ TIBPS in Solvesso 100 or kerosene.

a $1 \mathrm{M}$, el $\mathrm{NaCl}$ tiende a precipitar a esta temperatura de $25^{\circ} \mathrm{C}$.

Cuando el medio $\mathrm{NaCl} 4 \mathrm{M}$ se cambia totalmente por otro $\mathrm{HCl} 4 \mathrm{M}$, el equilibrio de extracción de $\mathrm{Ag}(\mathrm{I})$ por el STIBF también se alcanza rápidamente, aproximadamente a los $5 \mathrm{~min}$ de agitación, no aumentando, como en el caso anterior, el porcentaje de extracción de $\operatorname{Ag}(\mathrm{I})$ para tiempos de contacto más prolongados. Las fases orgánicas contenían $100 \mathrm{~g} / \mathrm{l}$ STIBF en queroseno y las fases acuosas eran de $0,05 \mathrm{~g} / \mathrm{l} \mathrm{Ag}(\mathrm{I})$ en el medio $\mathrm{HCl} 4 \mathrm{M}$. Para este caso,

TABLA III.- Influencia del medio acuoso en la extracción de $\mathrm{Ag}(\mathrm{I})$ mediante STIBF

TABLE III.- Influence of the aqueous media on Ag(I) extraction by TIBPS

\begin{tabular}{|c|c|c|}
\hline $\begin{array}{c}\mathrm{HCl}, \\
\mathrm{mol} / \mathrm{l}\end{array}$ & $\begin{array}{c}\text { STIBF, } \\
\mathrm{g} / \mathrm{l}\end{array}$ & $\begin{array}{c}\text { Extrac. } \mathrm{Ag}(\mathrm{I}), \\
\%\end{array}$ \\
\hline- & 25 & 49,2 \\
0,5 & 25 & 36,0 \\
- & 50 & 80,2 \\
0,5 & 50 & 75,3 \\
1,0 & 50 & 76,0 \\
\hline
\end{tabular}

Fase acuosa: 0,2 g/l Ag(I) y $\mathrm{NaCl} 4 \mathrm{M}$. Fase orgánica: STIBF en queroseno. Temperatura: $25^{\circ} \mathrm{C}$. la temperatura fue de $20^{\circ} \mathrm{C}$, y los tiempos de agitación estaban comprendidos entre 1 y $30 \mathrm{~min}$.

La figura 3 muestra el efecto que el cambio de temperatura tiene sobre la extracción de este metal. Las disoluciones ensayadas fueron las mismas que las mencionadas anteriormente, mientras que el tiempo de agitación fue de $10 \mathrm{~min}$.

Se observa que, en este medio, el aumento de la temperatura también da lugar a una disminución de la extracción de plata; la comparación de los resultados obtenidos con los que se consiguen en el medio acuoso $\mathrm{NaCl} 4 \mathrm{M}$, permite deducir que para cada temperatura ensayada en el medio $\mathrm{HCl}$ la extracción del metal es mayor que en el medio $\mathrm{NaCl}$; la diferencia de los resultados se hace mayor a medida que aumenta la temperatura a la que se lleva a cabo la extracción.

En este medio $\mathrm{HCl} 4 \mathrm{M}$, la reacción de extracción de la plata por el STIBF también presenta carácter exotérmico, según se deduce del valor de $-41,9 \mathrm{~kJ} / \mathrm{mol}$ correspondiente al cambio de entalpía de la reacción.

\subsection{Isotermas de equilibrio de extracción de $\operatorname{Ag}(\mathrm{I})$ y mecanismo de la reacción}

Las isotermas de extracción se obtuvieron a partir de fases orgánicas que presentaban distintas

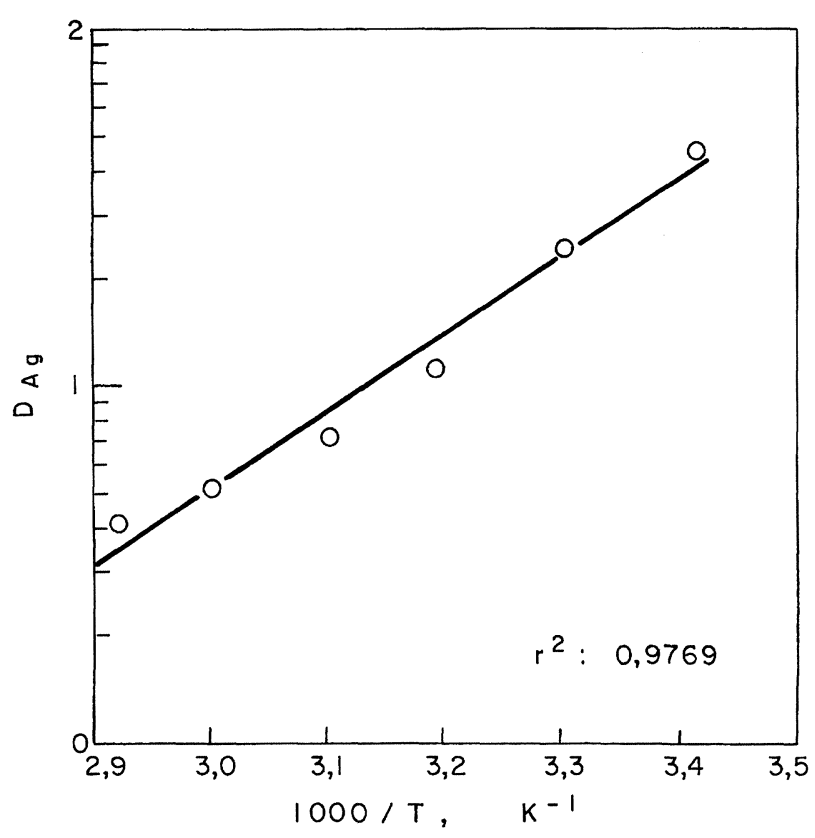

FIg. 3.- Variación del $\log D_{\mathrm{Ag}}$ frente a $1.000 / T$ para la extracción de plata. Fase acuosa: $0,05 \mathrm{~g} / 1$ $\mathrm{Ag}(\mathrm{I})$ en medio $\mathrm{HCl} 4 \mathrm{M}$. Fase orgánica: $100 \mathrm{~g} / \mathrm{l}$ STIBF en queroseno.

FIG. 3.- Arrhenius plot for the extraction of $\mathrm{Ag}(\mathrm{I})$ by TIBPS. Aqueous phase: $0.05 \mathrm{~g} / \mathrm{l} \mathrm{Ag}(\mathrm{I})$ in $\mathrm{HCl} 4 \mathrm{M}$ media. Organic phase: $100 \mathrm{~g} / \mathrm{l}$ TIBPS in kerosene. 
concentraciones de STIBF en queroseno y fases acuosas con distintas concentraciones de $\mathrm{Ag}(\mathrm{I})$ en un medio $\mathrm{NaCl} 4 \mathrm{M}$. La temperatura fue de $25^{\circ} \mathrm{C}$ y el tiempo de contacto de $10 \mathrm{~min}$. En la figura $4 \mathrm{se}$ ofrecen los resultados obtenidos.

El sulfuro de tri-isobutil fosfina es un agente de extracción por solvatación, por lo que la reacción de extracción de la plata se puede representar mediante:

$X_{(\mathrm{n}-\mathrm{m})}^{+} \mathrm{AgCl}_{\mathrm{n}_{\mathrm{ac}}}^{-(\mathrm{n}-\mathrm{m})}+p L_{\text {org }} \rightleftharpoons X_{(\mathrm{n}-\mathrm{m})} \mathrm{AgCl}_{\mathrm{n}} L_{\mathrm{Porg}}$

Dependiendo del complejo de plata predominante en la disolución acuosa, el valor de $n-m$ puede ser variable (10-12). Asumiendo la existencia de los complejos con $n-m$ igual a 2 ó 3 , más estables en este medio, el valor de $n$ sería entonces, respectivamente, de 4 ó 3 . Donde $L$ representa el agente de extracción, $X$ un catión y ac y org las fases acuosa y orgánica, respectivamente.

A partir de esta ecuación se obtiene que:

$$
K_{\mathrm{ext}}=\frac{\left[X_{(\mathrm{n}-\mathrm{m})} \mathrm{AgCl}_{\mathrm{n}} L_{\mathrm{p}}\right]_{\mathrm{org}}}{\left[X_{(\mathrm{n}-\mathrm{m})}^{+} \mathrm{AgCl}_{\mathrm{n}}^{-(\mathrm{n}-\mathrm{m})}\right]_{\mathrm{ac}}[L]_{\text {org }}^{\mathrm{p}}}
$$

y por definición del valor del coeficiente de distribución, se llega a la expresión final:

$$
\log D_{\mathrm{Ag}}=\log K_{\mathrm{ext}}+p \log [L]_{\mathrm{org}}
$$

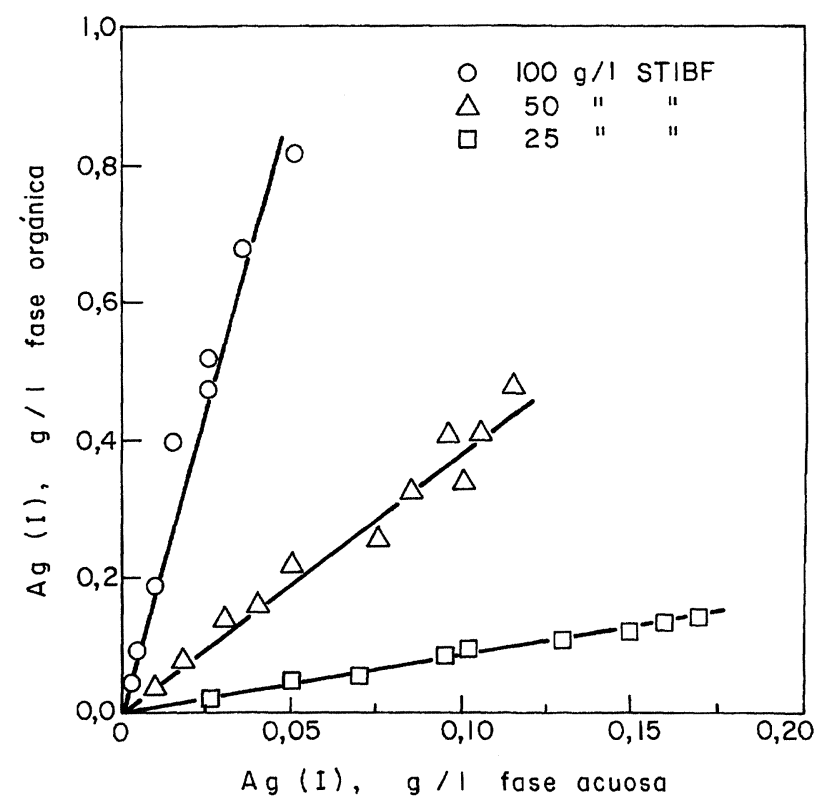

FIG. 4.- Isotermas de equilibrio de extracción de $\mathrm{Ag}(\mathrm{I})$ mediante STIBF.

FIG. 4.- Equilibrium loading isotherms for $\mathrm{Ag}(\mathrm{I})$ extraction by TIBPS.
El valor del coeficiente $p$ se puede calcular a partir de la representación de $\log D_{\mathrm{Ag}}$ frente a $\log$ $[L]_{\text {org }}$; la figura 5 muestra los resultados obtenidos deduciéndose que el valor de $p$ es igual a 2. Además, el hecho de que los valores de $\log D_{\mathrm{Ag}}$ sean prácticamente independientes de la concentración inicial de plata, y considerando cada concentración del agente de extracción, indica que en la fase orgánica no se forman especies o complejos polinucleares de $\operatorname{Ag}(\mathrm{I})$ (13).

A partir de los datos experimentales de la figura 5 , se deduce que el valor de $\log K_{\text {ext }}$, para la reacción representada en la ecuación [2], es igual a 2,05 .

Obtenido este valor gráficamente, los valores experimentales fueron, además, tratados numéricamente usando el programa LETAGROP-DISTRB (14), con el fin de confirmar tanto el modelo propuesto como el valor de $\log K_{\text {ext }}$.

En este programa se busca la mejor serie de constantes de formación que minimiza la suma de los errores al cuadrado, $U$, según:

$$
U=\sum\left(\log D_{\exp }-\log D_{\text {cal }}\right)^{2}
$$

en esta ecuación, $D_{\text {exp }}$ representa los coeficientes de distribución de la plata obtenidos experimentalmente, y $D_{\text {cal }}$, los valores correspondientes calculados por el programa teniendo en cuenta los

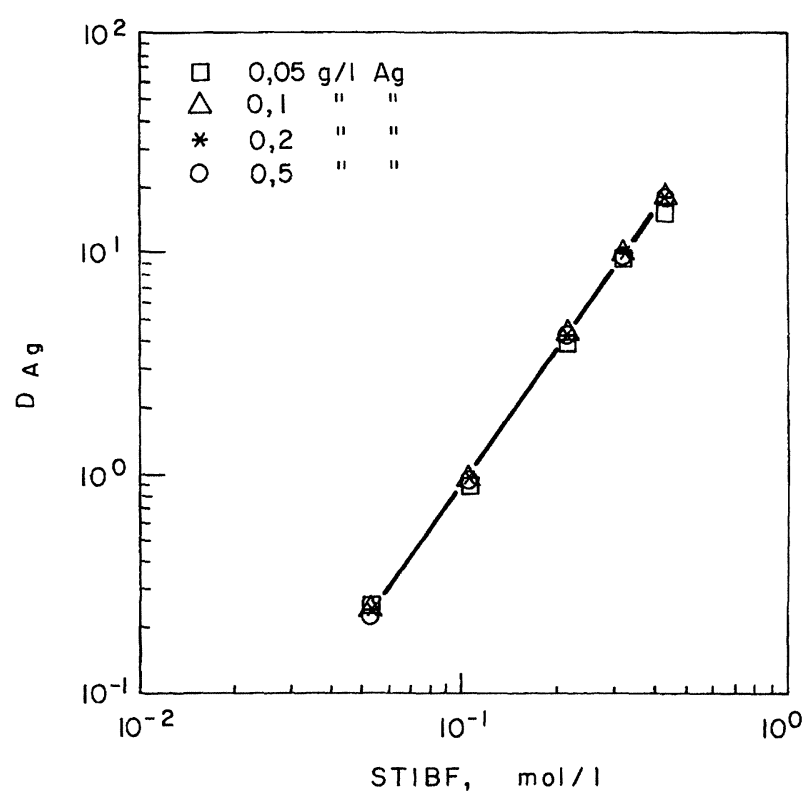

FIG. 5.- Variación de $\log D_{\mathrm{Ag}}$ frente a $\log$ $[\mathrm{STIBF}]_{\text {org }}$ para distintas concentraciones de plata. Fase acuosa: $\mathrm{Ag}(\mathrm{I})$ en medio $\mathrm{NaCl} 4 \mathrm{M}$. Fase orgánica: STIBF en queroseno. Temperatura: $25^{\circ} \mathrm{C}$.

FIG. 5.- Variation of $\log \mathrm{D}_{\mathrm{Ag}}$ vs. $\log [\text { TIBPS }]_{\mathrm{org}}$ at different initial silver concentrations. Aqueous phase: $\mathrm{Ag}(\mathrm{I})$ in $\mathrm{NaCl} 4 \mathrm{M}$ media. Organic phase: TIBPS in kerosene. Temperature: $25^{\circ} \mathrm{C}$. 
determinados modelos químicos y las constantes de formación en las dos fases líquidas. El valor de log $K_{\text {ext }}$, obtenido numéricamente, fue $2,0003 \pm 0,0511$, con $U$ igual a $0,8627 \cdot 10^{-2}$.

Para este sistema de extracción, $\Delta G^{\circ}$ es de $-11,35 \mathrm{~kJ} / \mathrm{mol}$, y $\Delta S^{\circ}$ es $-0,099 \mathrm{~kJ} / \mathrm{mol}$ a $25^{\circ} \mathrm{C}$.

\subsection{Selectividad del sistema Ag-STIBF frente a otros metales en disolución}

Junto a la plata pueden estar presentes en las disoluciones acuosas otros metales susceptibles de ser extraídos por el sulfuro de tri-isobutil fosfina. Se ha estudiado la selectividad del presente sistema de extracción frente a dos iones metálicos que, como el $\mathrm{Cu}(\mathrm{II})$ y el $\mathrm{Zn}(\mathrm{II})$, pueden acompañar a la plata en disolución.

Los ensayos experimentales se llevaron a cabo con disoluciones orgánicas de $50 \mathrm{~g} / \mathrm{l} \mathrm{STIBF}$ en queroseno y disoluciones de $5 \mathrm{~g} / \mathrm{l} \mathrm{Cu}$ (II) o $\mathrm{Zn}$ (II) en distintos medios acuosos. La temperatura fue de $25^{\circ} \mathrm{C}$ y el tiempo de agitación de $10 \mathrm{~min}$.

La tabla IV muestra los resultados obtenidos, donde se observa que en todos los casos la plata se extrae preferentemente a estos dos cationes metálicos; considerando los factores de separación experimentalmente obtenidos (mayores que 1), se deduce que es posible separar la plata del $\mathrm{Cu}(\mathrm{II})$ y del $\mathrm{Zn}$ (II), en este medio cloruro, empleando dicho agente de extracción.

Otros iones metálicos preciosos, como el $\mathrm{Au}(\mathrm{III})$ y el $\mathrm{Pd}(\mathrm{II})$, sí son extraídos por este agente, sobre todo en medio $\mathrm{HCl}(7,15$ y 16$)$.

\subsection{Estabilidad del sulfuro de tri-isobutil fosfina}

$\mathrm{Al}$ estar en contacto con distintas fases acuosas y debido a la propia composición de este agente de extracción, es posible que exista una degradación del mismo, posiblemente al correspondiente óxido. En este trabajo se ha evaluado esta posibilidad poniendo en contacto durante tiempos prolongados fases orgánicas de $50 \mathrm{~g} / \mathrm{l} \mathrm{STIBF}$ en queroseno con distintas disoluciones acuosas. La temperatura fue de $25^{\circ} \mathrm{C}$ y la relación de volúmenes $O / A=1$. En la tabla $\mathrm{V}$ se resumen las condiciones ensayadas.

Después de cada experiencia, las correspondientes fases orgánicas se pusieron en contacto con una disolución acuosa de $0,2 \mathrm{~g} / \mathrm{l} \mathrm{Ag}(\mathrm{I})$ en medio $\mathrm{NaCl}$ $4 \mathrm{M}$ a $25^{\circ} \mathrm{C}, 10$ min de agitación y relación $O / A=1$. Los resultados obtenidos se muestran en la tabla V. Dichos resultados se compararon con los obtenidos con una disolución recientemente preparada de 50 $\mathrm{g} / \mathrm{l} \mathrm{STIBF}$ en queroseno y la misma disolución acuosa mencionada con anterioridad. En este último caso, se obtiene un valor de $D_{\mathrm{Ag}}$ igual a 4,0 , que corresponde a un $80,2 \%$ de extracción del metal, por lo que la comparación con los valores obtenidos en la tabla $\mathrm{V}$ indica que en las condiciones experimentales ensayadas no tiene lugar una degradación o una pérdida apreciable de la capacidad de carga del sistema sulfuro de tri-isobutil fosfina-queroseno.

\subsection{Reextracción}

La reextración de la plata de la fase orgánica se ha abordado empleando una disolución estabilizada de tiosulfato sódico (3 y 7). La fase orgánica de 25 g/l STIBF en queroseno estaba cargada con 0,098 TABLA V.- Estabilidad del STIBF frente a distintas disoluciones acuosas

TABLE V.- Stability of the TIBPS against different aquous solutions

\begin{tabular}{|c|c|c|}
\hline $\begin{array}{c}\text { Disoluciones } \\
\text { acuosas }\end{array}$ & $\begin{array}{c}\text { Tiempo de } \\
\text { contacto, } \mathrm{h}\end{array}$ & $\begin{array}{c}\text { Extracción } \\
\mathrm{Ag}(\mathrm{I}), \%\end{array}$ \\
\hline $3 \mathrm{M} \mathrm{H}_{2} \mathrm{SO}_{4}$ & 300 & 79,0 \\
$2 \mathrm{M} \mathrm{HCl}$ & 225 & 81,1 \\
$15 \mathrm{~g} / \mathrm{F} \mathrm{Fe}(\mathrm{III})$ & 150 & 81,3 \\
$5,0 \mathrm{~g} / \mathrm{l} \mathrm{Cu}$ (II) & 150 & 80,0 \\
\hline
\end{tabular}

TABLA IV.- Selectividad del sistema STIBF-Ag(I) en medio $\mathrm{NaCl}$ concentrado frente a $\mathrm{Cu}$ (II) y $\mathrm{Zn}$ (II)

TABLE IV.-Selectivity of the TIBPS-Ag(I) system in concentrated $\mathrm{NaCl}$ media against to $\mathrm{Cu}(\mathrm{II})$ and $\mathrm{Zn}(\mathrm{II})$

\begin{tabular}{|c|c|c|c|c|c|}
\hline \multirow{2}{*}{$\mathrm{HCl}, \mathrm{mol} / \mathrm{l}$} & $D_{\mathrm{Cu}}$ & $D_{\mathrm{Zn}}$ & $D_{\mathrm{Ag}}$ & \multicolumn{2}{|c|}{ Factor de separación } \\
\cline { 5 - 6 } & & & $\mathrm{Ag} / \mathrm{Cu}$ & $\mathrm{Ag} / \mathrm{Zn}$ \\
\hline- & 0,12 & 0,11 & 4,0 & 33,3 & 36,4 \\
0,1 & 0,042 & 0,025 & 3,7 & 88,1 & 148,0 \\
0,25 & 0,029 & no extrae & 3,4 & 117,2 & - \\
0,5 & 0,018 & no extrae & 3,0 & 166,6 & - \\
\hline
\end{tabular}

Fase acuosa: 0,2 g/l Ag, 5,0 g/l Cu y Zn. Medio: $\mathrm{NaCl} 4 \mathrm{M}$. 
$\mathrm{g} / \mathrm{l} \mathrm{Ag}(\mathrm{I})$. El tiempo de contacto fue de $5 \mathrm{~min}$ y la temperatura de $25^{\circ} \mathrm{C}$; se emplearon distintas relaciones de fases $O / A$.

La correspondiente isoterma se muestra en la figura 6. Aunque es posible reextraer la plata, la manipulación y preparación de la disolución de reextracción no es fácil, siendo este uno de los principales inconvenientes de este sistema de extracción.

\section{CONCLUSIONES}

El sulfuro de tri-isobutil fosfina disuelto en queroseno es un agente de extracción efectivo de la plata en disoluciones concentradas de cloruro sódico, por ejemplo $4 \mathrm{M}$. La reacción de extracción es exotérmica, siendo el cambio de entalpía de $-40,9$ $\mathrm{kJ} / \mathrm{mol}$. El cambio de diluyente de la fase orgánica y el cambio del medio acuoso influyen en la extracción del metal. Así, con un diluyente aromático se mejora la extracción de plata con respecto a cuando se emplea un diluyente alifático. En el caso del diluyente aromático empleado, Solvesso 100, la reacción de extracción también es exotérmica, siendo $\Delta H^{\circ}=-31,0 \mathrm{~kJ} / \mathrm{mol}$. La adición de $\mathrm{HCl}$ al medio $\mathrm{NaCl} 4 \mathrm{M}$ hace que disminuya la extracción del metal; sin embargo, la sustitución del medio $\mathrm{NaCl}$ por $\mathrm{HCl} 4 \mathrm{M}$ produce el efecto contrario. En este caso la reacción también es exotérmica, el valor de $\Delta H^{\circ}$ es de $-41,9 \mathrm{~kJ} / \mathrm{mol}$.

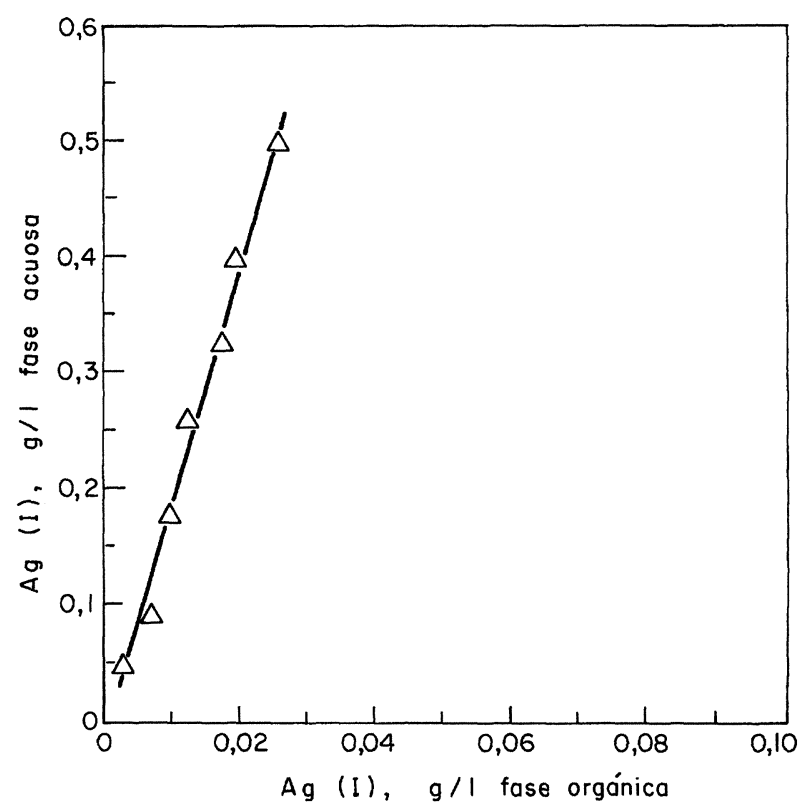

FIG. 6.- Isoterma de reextracción de Ag(I). Temperatura: $25^{\circ} \mathrm{C}$.

FIG. 6.- Ag(I) stripping isotherm. Temperature: $25^{\circ} \mathrm{C}$.
El sistema presenta buena selectividad con respecto a la presencia de otros iones metálicos tales como el $\mathrm{Cu}$ (II) y $\mathrm{Zn}$ (II) en la disolución acuosa. Asimismo, el sistema STIBF-queroseno es razonablemente estable frente a distintos medios acuosos.

El mecanismo de extracción de la plata en medio cloruro sódico concentrado por el STIBF se puede definir como una reacción por solvatación, con probable formación de la especie $X_{(\mathrm{n}-\mathrm{m})} \mathrm{AgCl}_{\mathrm{n}} L_{2}$ en la fase orgánica, en la que $L$ representa el agente de extracción. A partir de los datos experimentales, se obtiene que, para este sistema, $\log K_{\text {ext }}=2,0003 \pm 0,0511$ (numéricamente), mientras que $\Delta G^{\circ}$ es $-11,35 \mathrm{~kJ} / \mathrm{mol}$ y el valor de $\Delta S^{\circ}$ es de $-0,099 \mathrm{~kJ} / \mathrm{mol}$ a $25^{\circ} \mathrm{C}$.

\section{Agradecimiento}

Los autores agradecen a la Srta. M.I. Cuenca su ayuda en el trabajo experimental.

\section{REFERENCIAS}

(1) AlguACIL, F.J. Rev. Metal. En prensa, 1995.

(2) Alguacil, F.J. Rev. Metal. En revisión, 1994.

(3) Ritcey, G.M. y Ashbrook, A.W. Solvent extraction. Part I. Ed. Elsevier. Amsterdam, 1984: 87.

(4) Cox, M. Principles and practices of solvent extraction. Eds. J. Rydberg, C. Musikas y G.R. Choppin. Marcel Dekker Inc. Nueva York, 1992: 357.

(5) AMER, S. Rev. Metal. 19 (2), 1983: 96.

(6) PaIVA, A.P. y Abrantes, L.M. Solvent extraction in the process industries. Vol. 3. Eds. D.H. LogSDAIL y M.J. SLATER. Ed. Elsevier. Londres, 1993: 1.377.

(7) Cyanex 471X extractant. American Cyanamid Co. Nueva Jersey, 1984.

(8) Plazanet, C., Figueiredo, J.M., Alguacil, F.J. y col. Final Report ECC Contract MA2R-CT90-0003. European Community, 1993.

(9) Caravaca, C. Hydrometallurgy, 35, 1994: 53.

(10) Limpo, J.L., Luis, A., Galdós, W.G. y Hernández, A. Resúmenes de la 6 ${ }^{\mathrm{a}}$ Asamblea General del CENIM, Madrid, 1985.

(11) FrITZ, J. J. Soln. Chem., 14 (12), 1985: 865.

(12) Alguacil, F.J., Caravaca, C., Cobo, A. y Cuenca, M.I. Resúmenes de la 24 Reunión Bienal de la Real Sociedad Española de Química. Torremolinos, 1992: 417.

(13) Miralles, N., Sastre, A.M., Figuerola, E. y Martínez, M. Hydrometallurgy, 31, 1992: 1.

(14) LiEM, D.H. Acta Chem. Scand., 25, 1971: 1.521.

(15) Salvado, V., Hidalgo, M., Masana, A., Muñoz, M., Valiente, M. y Muhammed, M. Solvent Extr. Ion Exchange, 8 (3), 1990: 491.

(16) Martínez, S. y Alguacil, F.J. Hydrometallurgy. En revisión, 1995. 